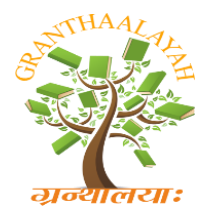

$$
\begin{aligned}
& \text { INTERNATIONAL JOURNAL OF RESEARCH - } \\
& \text { GRANTHAALAYAH } \\
& \text { A knowledge Repository }
\end{aligned}
$$

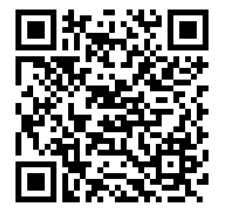

Management

\title{
DEVELOPING LIFE SKILLS IN YOUTH
}

\author{
Dr. A. Subramanian ${ }^{* 1}$ \\ ${ }^{* 1}$ Assistant Professor, Department of Education, University of Madras, Chennai-5, INDIA
}

\begin{abstract}
Life skills help young people navigate the challenges of everyday life. They enable them to develop into healthy, responsible, and productive adults. Adolescent life skills are central to psychological theories that aim to understand how skills and competencies develop. From a practical standpoint, the promotion of life skills has been identified as a key resource for enhancing positive and productive development in youth. As today's societies rapidly become ever more diversified both demographically and politically, our youth and adolescents face multifaceted challenges. What do these societal demands imply for the key skills that young people need to acquire? Answering this question is important not only for maintaining the quality of civic life and social cohesion, but also for enabling children and adolescents to develop into healthy, productive, and autonomous adults. Defining such skills can also improve our assessment of how well prepared young people are for life's challenges, and it can help us identify overarching goals for monitoring and evaluating education and intervention practices. Scholars, practitioners, and institutional administrators agree that having life skills help young people navigate these societal challenges, thereby contributing to their healthy, positive, and productive development. It is to define the key life skills in young people, identify their core domains, and review the theories and empirical evidence that address them and how they are acquired. The need for a developmental perspective is highlighted and the implications of a life skills framework for monitoring and evaluating educational and intervention practices are discussed. From the theoretical frameworks and exemplary models of life skills development in youth, it has become clear that despite conceptual differences, life skills frameworks for youth development suggest that all interventions need to provide age-appropriate ways for young people to fulfil their growth potential by improving their mental health, their learning, and their relationships with both adults and peers. On the other hand, this brief review has also shown that the frameworks provide a conceptual background for both research and application. They promise greater collaboration between researchers and practitioners, as well as improvements in developmentally informed intervention programs for youth. It highlights the potential for a new era of developmentally informed life skills intervention for youth. A coherent, rational, and sustainable intervention based on a youth development perspective and incorporating the principles of risk and resilience has tremendous potential for guiding the development of effective interventions.
\end{abstract}


Keywords:

Life Skills, Youth Development, Frameworks, Adolescents, WHO.

Cite This Article: Dr. A. Subramanian, "DEVELOPING LIFE SKILLS IN YOUTH" International Journal of Research - Granthaalayah, Vol. 4, No. 4: SE (2016): 65-74.

\section{INTRODUCTION}

Life skills help young people navigate the challenges of everyday life. They enable them to develop into healthy, responsible, and productive adults. Adolescent life skills are central to psychological theories that aim to understand how skills and competencies develop. From a practical standpoint, the promotion of life skills has been identified as a key resource for enhancing positive and productive development in youth. From the theoretical frameworks and exemplary models of life skills development in youth, it has become clear that despite conceptual differences, life skills frameworks for youth development suggest that all interventions need to provide age-appropriate ways for young people to fulfil their growth potential by improving their mental health, their learning, and their relationships with both adults and peers. On the other hand, this brief review has also shown that the frameworks provide a conceptual background for both research and application. They promise greater collaboration between researchers and practitioners, as well as improvements in developmentally informed intervention programs for youth. It highlights the potential for a new era of developmentally informed life skills intervention for youth. A coherent, rational, and sustainable intervention based on a youth development perspective and incorporating the principles of risk and resilience has tremendous potential for guiding the development of effective interventions.

\section{DEVELOPING LIFE SKILLS IN YOUTH}

As today's societies rapidly become ever more diversified both demographically and politically, our youth and adolescents face multifaceted challenges. What do these societal demands imply for the key skills that young people need to acquire? Answering this question is important not only for maintaining the quality of civic life and social cohesion, but also for enabling children and adolescents to develop into healthy, productive, and autonomous adults. Defining such skills can also improve our assessment of how well prepared young people are for life's challenges, and it can help us identify overarching goals for monitoring and evaluating education and intervention practices.

Scholars, practitioners, and institutional administrators agree that having life skills help young people navigate these societal challenges, thereby contributing to their healthy, positive, and productive development. It is to define the key life skills in young people, identify their core domains, and review the theories and empirical evidence that address them and how they are acquired. The need for a developmental perspective is highlighted and the implications of a life skills framework for monitoring and evaluating educational and intervention practices are discussed. 


\section{WHAT ARE LIFE SKILLS?}

In an effort to identify the skills needed to maintain the quality of civic live, a successful and meaningful personal life, and positive social relationships, authors have proposed various definitions and conceptualizations of life skills. The World Health Organization (1997) defines youth as people between the ages of 15 and 24 years and adolescents as people between the ages of 10 and 19 years. The term "young people" can be used as shorthand for these groups combined (ages 10 to 24). In general, life skills are considered to be those abilities that help promote well-being, positive health outcomes, and productive development. They comprise a set of core skills that empower young people to take positive steps to promote health, positive social relationships, and positive contributions to society. The question is what young people must have to function well in society as they find it. Life skills are important in helping them shape their world, not just cope with it. Thus, the concept of life skills transcends previous concepts such as coping and adaptation to circumstances; it presupposes an active, autonomous, and responsible stance towards the self in the social world.

According to the World Health Organization (1997), life skills are abilities for adaptive and positive behaviour that enable individuals to deal effectively with the demands and challenges of everyday life. By emphasizing adaptation and a positive orientation, this commonly used definition is consistent with the skills-based, positive approach to youth development. The PanAmerican Health Organization defines life skills as social and interpersonal skills (including communication, refusal skills, assertiveness and empathy), cognitive skills (including decisionmaking, critical thinking, and self-evaluation), and emotional coping skills (including stress management and increasing an internal locus of control). UNICEF (2002) defines life skills as psychosocial and interpersonal skills that help people make informed decisions, communicate effectively, and develop the coping and self-management skills needed for a healthy and productive life. Life skills can be applied to actions directed at either the self, other people, or the local environment; their goal is to promote health and wellbeing at all these levels.

The Organisation for Economic Cooperation and Development (OECD) likewise emphasizes the necessity of good life skills for achieving the outcomes desired by both society and its individual members, who must cope with important societal demands in a wide variety of contexts. Life skills are important for everybody. In contrast to the previously cited definitions, the OECD definition emphasizes "competence" rather than "skills." It implies that competence is more than just skills; it is also the ability to cope with complex demands by drawing on and mobilizing all of one's psychosocial resources (including skills) in a given context. Thus, life skills include not only behaviour, but also attitudes and knowledge. The OECD specifies three key competencies.

Other definitions of life skills follow the OECD definition in strongly emphasizing the ability to balance different types of skills, which include behaviour, cognitions, values, attitudes, and emotions. Thus, these definitions encompass the whole panoply of skills that individuals need for well-being and productive development. For example, UNICEF (2001) defines life skills as a behaviour development approach designed to address a balance of three areas: knowledge, attitude and skills. The ability to coordinate this interplay of various intrapersonal and interpersonal abilities means the difference between success and risk in dealing with the challenges of everyday life. In short, these definitions encompass a wide range of intra- and 
interpersonal skills that young people can use to integrate their knowledge, emotions, values, and actions to achieve specific social or academic goals and contribute to society. Some definitions also emphasize the need to consider spirituality as an important area in which life skills are embedded.

This framework defines not only the intra-personal and interpersonal skills needed to navigate the challenges of today's world, but also an educational policy that identifies core skills, a set of specific goals, and the support systems that young people need for positive development. Most definitions of life skills are rather broad and generic; they often include the term "livelihood skills," which refers to the basic abilities needed to pursue individual and household economic goals. Although addressing life skills would be meaningless if how one earns a living were not taken into account, livelihood skills alone are not enough for achieving long-term change; young people also need the higher-order intra- and interpersonal skills discussed above.

Because the above definitions are meant to apply to the general population, they are not developmentally oriented. If we are interested in young people, adaptation and positive outcomes must be defined developmentally. This means that the level of a given life skill needs to be considered in respect to a young person's age and in relation to his or her general biological, cognitive, emotional, and social development. For example, they have defined life skills as those competencies that enable young people to deal with the challenges of their lives in a manner that is adequate to their age and thus allows for healthy and positive development. Generally, people are considered to be skilled if they master the tasks that society considers appropriate for a person of that age or stage and that are inherent in human development. The WHO (1997) defines life skills as those skills and competencies that enable children and adolescents to deal adequately with their daily challenges and developmental tasks.

\section{THE CORE DIMENSIONS OF LIFE SKILLS}

Young people need a wide range of intra- and interpersonal skills to face the complex challenges of today's world, but it would be of little practical value to produce a long list of all the skills they may need in various contexts and at various times in their lives. A better choice is to consider only the skills that are most helpful to young people in managing situations in an age appropriate way. The preceding definition of life skills makes it clear that the core skills must not be chosen arbitrarily. Rather, their selection should be the result of carefully considering the individual and psychosocial prerequisites for a healthy and productive individual life and a well functioning society. Collectively, these core skills can be considered a loosely coupled system of different aspects of specific abilities. Their underlying unity is represented by a structural continuity of developmental changes. This framework implies that in any given individual, some skills are well developed and others poorly developed. In addition, contextual variables can influence how well a skill develops. Complexity is inherent in frameworks that are as broad and comprehensive as life skills, and this complexity has implications for measuring, monitoring, and evaluating life skills.

To answer the question of what skills young people need to cope with the changes in modern society, we must begin with a coherent concept of what constitutes the core skills. The framework must consist of a set of specific abilities bound together in an integrated whole. It is 
necessary for adaptation and healthy outcomes to balance different cognitive, emotional, and behavioural skills, as well as creative capacities and psychological resources such as attitudes, motives, and values. This ability to integrate different life skills crucially depends on how young people construct their social experiences, including their thoughts, emotions, and relationships with others, and how they integrate them into their self-concept. This task requires that they reach a level of social maturity that allows them to distance themselves from social pressures, adopt different perspectives, make independent judgements, and take responsibility for their actions. Adoption of this integrative framework has led to broad agreement on 10 to 12 specific intra- and interpersonal life skills that young people must have to deal effectively with the challenges they face in their lives in an age-appropriate and adaptive manner.

These core skills include abilities to make decisions, solve conflicts and problems constructively, think creatively, think critically, communicate effectively, be self-aware, deal with stress, regulate one's emotions, empathize with others, and establish and maintain good social relationships.

These core skills can be classified in four broad dimensions:

- Critical thinking and cognitive skills

- Coping skills and self-management

- Social and moral skills and

- Communication skills

Logically, it is possible and important to separate the intrapersonal and interpersonal levels. However, in everyday life, what happens inside a person and his or her relationships is continuously connected and exchanged.

The reflects a positive, asset-based view of young people's development, it is consistent with conceptualizations of youth development such as the risk and resiliency framework and the positive youth development perspective, which treats all adolescents as having strengths and potential. Scientists studying adolescent development have proposed the "Six Cs" of this development.

They are
- Competence
- Confidence
- Connection
- Character
- Caring and
- Contribution

They are a way to conceptualize positive youth development and integrate its various separate indicators, including self-esteem, empathy, and emotion regulation. Likewise, the developmental assets model incorporates a set of internal assets that promote positive youth development. They include commitment to learning, positive moral values, social competencies, and positive identity. The contribution to society and value components of life skills, in particular, have been underemphasized in the literature, despite their importance if life skills are to be conceptualized as including the ability to take responsibility for oneself and others. Any life skills framework 
must be anchored in a perspective that emphasizes the mutual and dynamic interaction between individuals and society. An emphasis on democratic values implies that adolescents need to be able to both achieve their developmental potential and contribute to a well-functioning society. This complementarity of individual and societal goals should be reflected in a framework of skills that acknowledges both individuals' autonomous development and their competent and responsible interactions with others.

A life skills framework places several requirements on young people in different contexts and different situations.

It specifies that the key life skills must

(a) Be of particular value

(b) Have multiple areas of usefulness and

(c) Be needed by everyone.

The first of these, that the skill should be valued as a fundamental resource of human capital, means that application of the skill should have measurable social benefits. Recent research reinforces the view that human capital brings key individual and social benefits such as better health, an improved sense of well-being, and increased civic engagement. The second condition, that skills should yield benefits and support in various contexts, means that they should apply in multiple areas of life. For example, some skills are needed in individuals' professional, personal, and civic lives, as well as to promote their health. It is such universal skills that meet the criterion of being core. The third condition, that core skills should be important for all young people, deemphasizes skills that are of use only in a specific occupation or within a specific group. Finally, both scholars and practitioners agree that core life skills are applied in a wide variety of social contexts and academic content areas. They also are critical to interventions such as drug abuse and HIV/ AIDS prevention, resilience enhancement, peace promotion, prevention of aggression and developmental disorders, career counselling, and many others.

It is important to keep in mind that the life skills definitions described above do not include external systems of support. Rather, the focus is on internal competencies and abilities, along with internal values and attitudes. This is in contrast to other theoretical accounts in the positive youth development tradition, which have conceptualized the dimensions of external support systems as a set of "assets" such as supportive relationships. Thus, this latter approach highlights the importance of social context in development.

\section{LIFE SKILLS FRAMEWORKS FOR YOUTH DEVELOPMENT}

The framework helps us understand the social cognitions, emotions, and motives that young people apply in constructing their reality at any given time. Furthermore, it helps the practitioner communicate with young people in ways that match their understanding of the social world, thereby allowing the practitioner to support their growth and thriving. The primary theories of adolescent development have most often evolved in the tradition of cognitive and psychosocial theories. These theories usually describe development in terms of sequences of increasing levels of complexity, maturity, and differentiation. Erikson's psychosocial theory describes the development of identity across the lifespan in socio-historic terms. Identity refers to feelings of self-trust that develop through our daily interactions with supportive others. At each stage, 
people experience a conflict typical of that stage, which serves as a turning point in their development. The successful mastery of this conflict, such as identity refinement in adolescence, helps to enhance strengths. In contrast, unresolved crises are assumed to cause disaffection and maladaptive outcomes. Many of the early developmental theories looked at adolescent development through the lens of a deficit model. During the later phases of theory development, and informed by an increasing number of empirical studies of adolescent development, theories have been increasingly influenced by the idea that adolescence is characterized by diversity, a systematic sequence of change and stability in development, and context dependence. Recent perspectives suggest that increases in well-being, thriving, and social contributions are possible for all adolescents if their strengths are accompanied by adequate social support.

In the following subsections, we briefly discuss the two main frameworks that emphasize life skills as the conceptual foundation for both adolescent development and prevention: (a) the positive youth development framework, and (b) the developmental risk and resiliency framework.

\section{THE POSITIVE YOUTH DEVELOPMENT FRAMEWORK}

The Positive Youth Development framework evolved from concepts grounded in developmental science, the systematic study of the processes that cause continuity and change over time in the bio-psychological characteristics of human beings. This approach aims to investigate and prevent risky behaviour by the enhancement of life skills, supportive relationships, and positive social conditions. Furthermore, it aims to promote thriving behaviour by young people integrating their social support systems. Research supports the link between positive development and both risk and thriving behaviour. More recently, the debate has shifted from how to enhance positive outcomes and decrease dysfunctional outcomes at the individual level to how to improve the settings and contexts of youths' daily lives in which these outcomes occur. Several frameworks have been developed to describe the elements of positive development in youth. There is consensus that positive youth development applies to all young people. Each young person has the inherent capacity for positive development and can benefit from supportive relationships.

This is true regardless of the context, examples of which are gender, age group, and ethnicity. In contrast, how positive youth development is applied can vary from one context to another. For example, empowerment is considered a developmental asset for all youth, but the strategies for promoting this asset can vary considerably as a function of social context.

Young people are considered the major agents in their own development, which means that they have the ability to create the supportive relationships and communities they need to grow and thrive. The positive youth development approach is thus holistic in the sense that it considers the community in relation to the whole child and the child as a full partner in this relationship. The set of key skills it incorporates refers not only to the development of one's personal identity, but also to one's contributions to and affiliation with civil society. Furthermore, positive youth development is a process that inherently occurs during the continual exchanges between individuals and a supportive social context. A positive developmental trajectory is established when youth participate in multiple relationships, programs, and institutions that promote their health and productive development. A wide range of empirical studies has supported the diverse 
and complex relations between various life skills, positive developmental outcomes, and problem behaviour. Recent research has increasingly focused on the trajectories of positive and negative development and their inter-relationships. Future research linking developmental trajectories of positive development and dysfunction with developmental processes and development by context interactions will lead the field of developmental science to a revised understanding of the richness of youth development.

\section{THE DEVELOPMENTAL RISK AND RESILIENCY FRAMEWORK}

The developmental risk and resiliency perspective is a comprehensive framework entailing the dynamic interplay between life skills, social context, and biological and neurological factors. Developmental scientists have elaborated in what sense skills, assets, and competencies are developmental and what that means for both a developmental theory and intervention in the risk and resiliency developmental framework and systems theory. This approach has also been emphasized in public health as a way to design successful social policies. It requires that the risks be conceptualized as developmental disorders emerging from adolescents' developmental history.

This history includes the adolescents' life experiences, the evolution of the meaning structures embedded in their social contexts, and their inherited predispositions. The development of aggressive behaviour is associated with particular social cognitions and emotions that individuals draw upon to construct their social world in each level of development. It is also crucial to this perspective that the human ability to change and recover, as well as to develop resiliency, be treated as a developmental capacity supported by the adoption of a developmentally differentiated approach. Although risks can turn into problems, they also can encourage the evolution of strengths, thriving, and resilience. Resiliency has been defined as the ability of an individual to develop and succeed despite adversity. It is not stable; instead, it continually evolves as individuals interact with their social environment. Because young people are active meaning makers who construct and invent their world, they can overcome previous traumas by giving new meaning to old events, self-reflecting, and exploring alternative thoughts and actions. Developmental scientists have identified two key developmental outcomes, both of which apply to multiple situations and developmental domains. The first is increased competence resiliency, which is demonstrated by the acquisition and further development of the knowledge or skills needed to guide one's behaviour. The second is increased dysfunction (i.e., risk), or the recurrent manifestation of difficulty in maintaining control and integrating one's actions. It is widely accepted that the association of positive and negative outcomes and developmentally rooted skills are intertwined and depend on differences in underlying developmental processes. The course of development depends on exposure, that is, contact between the developing person and the proximal processes in which the person engages. These, in turn, are rooted in the continual exchanges between the person, the person's environment, and the successive periods of social stability and change. In short, developmental outcomes must be understood as the result of a dynamic interplay between biological systems, human development, and social context. 


\section{THE CLOVER MODEL}

The clover model is the attempt to conceptualize the specific relations of development with both risks and resiliencies. Elaboration of the clover model has been guided by research, prevention practices, and the goal of creating a sound developmental theory. The model incorporates different levels of socio-cognitive, emotional, and social functioning, as well as the key vulnerabilities, risks, and symptoms that arise at each level of development in adolescence. Each developmental level causes new strengths to emerge and provides opportunities to rework past vulnerabilities. On the other hand, each of these new systems can also lead to new weaknesses or more complex versions of old dysfunctions. An increasing ability to be empathic and take the perspective of others risks a concurrent increase in depression. Studies support the view that the development of social cognition and empathy accompanies risks. Empathy has been shown to negatively relate to aggressive behaviour in adolescents, whereas identity and social-cognitive development is in part positively associated with symptoms of depression. The clover model also suggests that adolescents continually manifest each of the proposed different developmental levels and must find ways to balance them all. The clover model, as well as other risk and resiliency developmental frameworks, represents a holistic approach to adolescent development that can be used to plan developmentally informed interventions.

\section{EVALUATION EVIDENCE}

Developing and implementing effective intervention strategies involve understanding the skills that are the target of the invention. It is also necessary to understand when and how these skills manifest, as well as when and why a given problem behaviour or dysfunction occurs during the course of development. It is clear from the previous discussion that the intervention must be tailored to each adolescent's level of development. It is this skill or competence level that provides the key information on the adolescent's strengths and the social support systems that are necessary for the adolescent to grow and thrive. A mismatch between an adolescent's developmental capacities and a practitioner or program developer's perception of those capacities can have an adverse effect on intervention and/or mentoring programs.

\section{LESSONS LEARNT-IMPLICATIONS FOR MONITORING AND EVALUATION}

Any effective program that adopts a developmental perspective to promote life skills must be designed thoughtfully and systematically. Which life skill(s) to pick and which strategies to adopt in designing the intervention are crucial questions that must be answered if a program is to be successful. Thus, it is important to identify and describe the life skills that are essential in the given context and relevant to the issue(s) that will be addressed. It is helpful that the life skills frameworks have a broad enough scope to successfully address a wide range of such issues, including, for example HIV/AIDS prevention, violence prevention, and social exclusion.

Different issues and contexts require different life skills. The questions of the strategic design phase include then, which age groups are to be served and in what context is the program to be implemented. If a life skills intervention is to be effective, it must have an elaborate design that addresses implementation issues and provides concise and realistic methods for monitoring the implementation. Typically, intervention programs assure that all the various settings in which 
youth development takes place are involved in the process. It is necessary to demonstrate that the program can work under the proposed preconditions. The program must be able to achieve specified outcomes in feasible, sustainable, and affordable ways in real-world settings.

\section{CONCLUSION}

We have outlined an integrated set of specific developmentally adequate abilities that we have labelled "life skills for young people". These skills, which everyone needs, are useful in multiple domains and always play out in a social context. They are incorporated in developmental frameworks such as positive youth development and developmental risk and resiliency. They are genuinely strengths-oriented and conceptualize the role of the child as a partner with adults. They emphasize not only positive behavioural skills, but also the cognitions, emotions, attitudes, and values that come to the fore in different domains of development. Most importantly in this connection, they embody the recognition that certain methods and interventions are most effective at particular ages and levels of development. The gap between developmental theory and evidence-based intervention programs, however, is still not completely filled. An important goal for the future is the rigorous implementation of integrative concepts that systematically link developmental theory with prevention practices directed at young people. Foremost is the increasing social fragmentation that all today's youth's must navigate, wherever they live in the world. Not only does each social context present its own significant challenges, such as the loss of family structure or widespread bullying in schools, but there is also a lack of coordination between social systems and how they are related to one another. This is lack of coordination has led to increasing fragmentation, or as some have labelled it, "chaos". Despite the efforts of intervention programs, research over the past four decades reveals a progressive decline in concern for the well-being of others - expressed primarily not in words but in actions.

\section{REFERENCES}

[1] Benson, P. L. (2007). Developmental assets: An overview of theory, research, and practice. In R. K. Silbereisen \& R. M. Lerner (Eds.), Approaches to positive youth development (pp. 33-58). Thousand Oaks, CA: Sage.

[2] Benson, P. L., \& Scales, P. C. (2009). Positive youth development and the prevention of youth aggression and violence. European Journal of Developmental Science, 3, 218-234.

[3] Benson, P. L., Scales, P. C., Hamilton, S. F., \& Semsa, A., Jr. (2006). Positive youth development: Theory, research, and applications. In R. M. Lerner (Ed.), Handbook of Child Psychology: Vol. 1: Theoretical models of human development (pp. 894-941). Hoboken, NJ: Wiley.

[4] Larson, R. (2006). Positive youth development, willful adolescents, and mentoring. Journal of Community Psychology, 34, 677-689.

[5] Hamilton, S.F., Hamilton, M. A., \& Pittman, K. (2004). Principles for youth development. In S. F. Hamilton, \& M. A. Hamilton, (Eds.), The youth development handbook: Coming of age in American communities (pp. 3-22). Thousand Oaks, CA: Sage.

[6] Connell, J. P., Gambone, M. A., \& Smith, T. J. (2000). Youth development in community settings: Challenges to our field and our approach. Youth Development: Issues, Challenges and Directions (pp. 281-300). Philadelphia: Public/Private Ventures. 